\title{
Laboratory and natural heritabilities of male courtship song characters in Drosophila montana and $D$. littoralis
}

\author{
J. ASPI \& A. HOIKKALA \\ Department of Genetics, University of Oulu, SF-90570, Oulu, Finland
}

\begin{abstract}
We estimated heritabilities for several male courtship song characters in two Drosophila species using father-son regression under conditions where both fathers and sons had been raised in the laboratory. In $D$. montana the heritabilities of song characters were rather high $(-0.23$ to 0.80$)$ and in most cases significant. In $D$. littoralis the heritabilities of song characters were generally lower $(-0.33$ to 0.18$)$, and none of them was significantly larger than zero. We also estimated heritabilities regressing characters of wild-caught fathers with those of their laboratory reared sons, and used the method employed by Riska $e t$ al. to estimate the lower bound of heritabilities in nature. In $D$. montana most and in $D$. littoralis all of the across-environment heritabilities were non-significant ( -0.15 to 0.43 and -0.04 to 0.15 , respectively), and in some cases the across-environment heritabilities were significantly lower than the heritabilities measured under laboratory conditions. The low across-environment heritabilities appeared to be due to larger phenotypic variability of song characters in the field and in some cases also due to genotype-environment interactions.
\end{abstract}

Keywords: courtship songs, Drosophila, environmental variation, genotype-environment interaction, heritability, sexual selection.

\section{Introduction}

The basic assumption used in the two groups of sexual selection models of evolution of female choice is the heritability of the preferred male trait (e.g. Bradbury \& Andersson, 1987). Existence of heritable variation in preferred male characters has been demonstrated in several insect studies under conditions designed to minimize environmental variation (Cade, 1981; Carson \& Lande, 1984; Hedrick, 1988; Moore, 1990). The heritability of male characters has, however, only rarely been studied in field conditions (Møller, 1989, 1991).

Genetic parameters estimated for male characters under laboratory conditions are often extrapolated to concern these characters also in the field. The ratio of additive genetic variation to the total phenotypic variation can, however, be quite different in the field, where the additive variation in preferred male characters could be masked completely by larger environmental variation (e.g. McLain, 1987; Riska et al., 1989; Hoffman, 1991). Several insect studies have demonstrated that phenotypic variation in quantitative characters can be several fold larger among individuals caught in the wild than among the individuals reared under labora- tory conditions (e.g. Coyne \& Beecham, 1987; Ruiz et $a l ., 1991)$. Differences between laboratory and natural environments may also cause genotype-environment interaction, which can further inflate the genetic variances and bias estimates of heritabilities and genetic correlations (e.g. Riska et al., 1989; Hoffman, 1991).

Prout (1958) has employed a method to examine the heritability of quantitative traits in the field by correlating phenotypic characters of offspring raised in the laboratory with those of their parents raised in the natural environments. Similar approaches have been used in several other studies (Coyne \& Beecham, 1987; Prout \& Barker, 1989; Hoffman, 1991). In an appendix to Coyne \& Beecham (1987), Lande has shown that the regression of laboratory-reared offspring on wild-caught parents can be used as a reliable estimate of heritability in the natural environment only when the additive genetic variance is similar in different environments. Lande also showed that this condition is met when the estimated additive genetic variance in the laboratory is equal to the estimated covariance between offspring in the laboratory and midparent in nature, and that the regression across 
environments sets the lower bound to the heritability if additive genetic variance exceeds the covariance. Riska et al. (1989) extended Lande's approach to show how a lower bound on the heritability in nature can often be estimated even when Lande's condition is not met.

We report here the measurements of the heritable variation in characteristics of the courtship songs of two Drosophila species, D. montana and D. littoralis, under laboratory and field conditions using the approach of Riska et al. (1989). Some of these song characters are known to be influenced by sexual selection in the field (Aspi \& Hoikkala, in preparation).

\section{Materials and methods}

\section{Flies used}

The wild-caught males were derived from a natural population near the town of Kemi in northern Finland $\left(65^{\circ} 40^{\prime} \mathrm{N}, 23^{\circ} 35^{\prime} \mathrm{E}\right)$ during the breeding season in May 1989. The flies were collected from the surface of malt-baited traps with the aid of a collecting net and a pooter. The collection of flies is provided in detail in Aspi et al. (1993).

We carried out two experiments to obtain the heritability estimates 'within laboratory' and 'across the environments' for male song characters. Males used in the first experiment were of the first and the second generation offspring of wild-caught flies. All these males had been raised in the laboratory. In the second experiment we used wild-caught males and their laboratory-reared sons. Females were collected for both experiments from a population cage stock initiated the previous summer with the progeny of 30 wild-inseminated females from the Kemi population.

The sires were mated with virgin females. Throughout the experiment the females were allowed to oviposit $50 \pm 10$ eggs per vial to avoid overcrowding. The emerging male progeny were aged in vials until they were sexually mature (about 3 weeks). The songs of the father and one of his sons were recorded and analysed.

\section{Song recordings and analysis}

The songs were recorded in the laboratory while the males were courting virgin sexually mature conspecific females in a plastic net-covered petridish (diameter 50 $\mathrm{mm}$, height $12 \mathrm{~mm}$ ). Song recordings were made with a JVC condenser microphone and a Sony TC-FX 33 cassette recorder. Oscillograms of the male songs were analysed by a Gold 1425 digital oscilloscope.

The song of $D$. montana consists of pulse trains (Fig. 1). The means for males of this species were calculated over the song characters in five pulse trains. For each pulse train we measured the length of the pulse train (henceforth PTL), the length of fourth sound pulse of each pulse train (PL) and the distance from the beginning of this pulse to the beginning of the next one (IPI). We also counted the number of pulses in a pulse train $(\mathrm{PN})$ and the number of cycles in the fourth sound pulse (CN). The basic frequency (FRE) for each pulse train was calculated as $1000 /(\mathrm{PL} / \mathrm{CN})$. The song of $D$. littoralis consists of discrete sound pulses (Fig. 1). For the males of this species the mean PL, IPI, CN and FRE were measured or counted over their five successive sound pulses. For the statistical analysis PTL, IPI and PL were transformed to natural logarithms in both species.

Song character measurements were based on only one recording per male, and so the repeatability of characters could not be estimated. To get an impression on how much the songs vary within males, we estimated the repeatabilities of the songs from the data collected during the description of songs of $D$. virilis group species (Hoikkala et al., 1982). These analyses included three $D$. montana and four $D$. littoralis laboratory strains. The songs of five males per strain were recorded in 3 subsequent days. The repeatabilities of song characters were estimated as the ratio of among-individual variance to the total variance and it sets an upper limit for the heritability of a character (Falconer, 1981). Confidence limits for the repeatabilities were estimated according to Bogoy \& Becker (1963), and the square root of the sampling variance of the intraclass correlation was used as approximate standard error of the estimates (Becker, 1975).

\section{Heritability estimates}

The heritabilities of the male song characters were estimated two ways: using father-son regression under conditions where both the fathers and the sons had been raised in the laboratory $\left(h^{2}\right.$ (SL-FL) $)$ and by regressing the characters of fathers collected in nature with those of their laboratory reared sons $\left(h^{2}(\mathrm{SL}-\mathrm{FN})\right)$. The heritabilities were estimated in both experiments as twice the slope of the regression of offspring character against paternal values.

Possible reasons for the differences between the heritability estimates measured within the laboratory and across the environments are the larger environmental variation in song characters among the wildcaught fathers and gene-environment interactions. The indication of the importance of larger environmental variation can be obtained by comparing the heritability estimate obtained from the across-environment regres$\operatorname{sion}\left(h_{(\mathrm{SL}-\mathrm{FN})}^{2}\right)$ and the ratio of additive genetic variance 

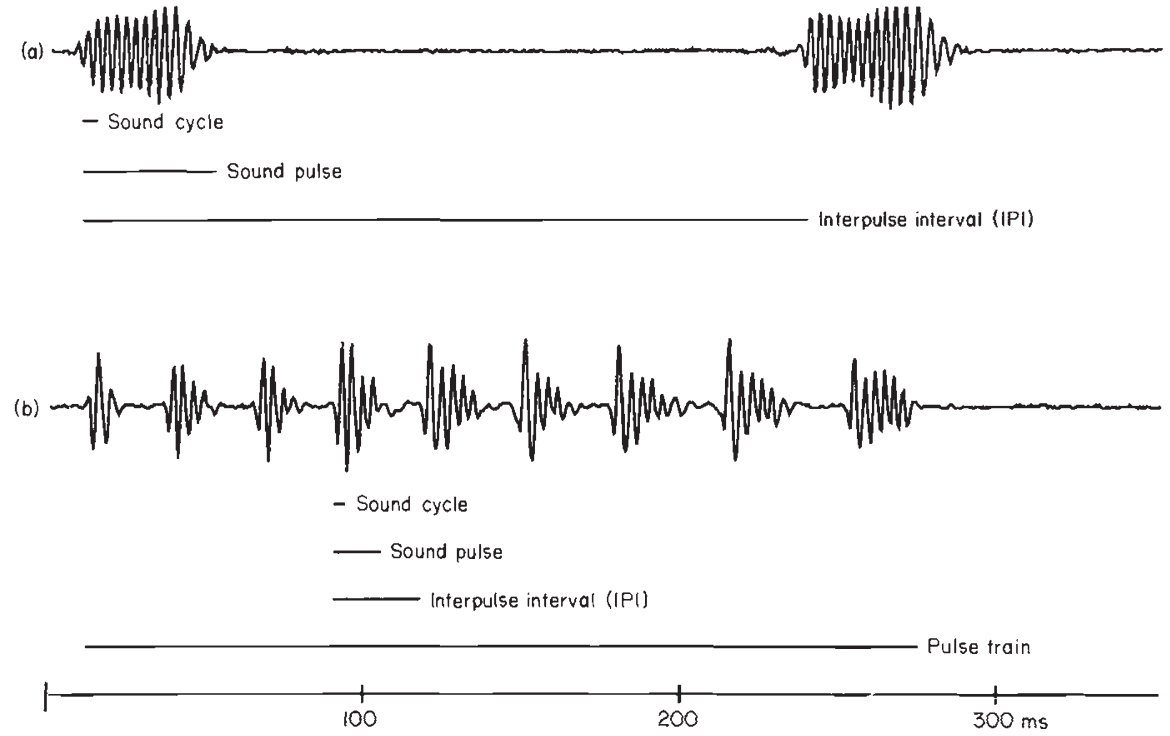

Fig. 1 Oscillograms of courtship songs of wild-caught males in D. littoralis (a) and in D. montana $(\mathrm{b})$. in the laboratory over the phenotypic variance in the wild $\left(V_{\mathrm{AL}} / V_{\mathrm{PN}}\right)$. This ratio provides an estimate of heritability $\left(h_{\mathrm{V}}^{2}\right)$ when the additive genetic variance in the field and in the laboratory is similar. If the estimate of $h_{\text {(SL-FN) }}^{2}$ exceeds the value of $h_{\mathrm{V}}^{2}$ then the additive genetic variance is larger in nature than in the laboratory setting the lower bound to the heritability in nature $\left(h_{\mathrm{MIN}}^{2}\right)$. If, on the other hand, the ratio of these parameters is in the opposite direction then the estimate of the lower bound of the heritability in nature is set as $4 b_{(\mathrm{SL}-\mathrm{FN})}^{2}\left(V_{\mathrm{PN}} / V_{\mathrm{AL}}\right)$ (Riska et al., 1989).

\section{Results}

\section{Repeatability}

The repeatabilities of IPI, CN and FRE were high in both species (Table 1). The repeatability of PL was also high in D. montana but not in $D$. littoralis. The proportion of total variation due to individual differences was non-significant only for PL in $D$. littoralis, but both PTL and PN had low repeatability and did not vary significantly among males in $D$. montana. To test if the low repeatability of these song characters was due to a reduced genetic variance in the old laboratory strains used for the repeatability measurements, we compared the variances in these strains and among the laboratory reared $F_{1}$ males. The analyses revealed significantly lower variances within each laboratory strain compared with the variation among the $F_{1}$-offspring males $\left(F^{\prime}\right.$-test) in PL in $D$. littoralis (indicating that the repeatability estimate may not be very representative for natural populations) but not in PTL and PN of $D$. montana.
Table 1 Repeatability of song character measurements among $D$. littoralis $(N=20)$ and $D$. montana $(N=15)$ males

\begin{tabular}{|c|c|c|c|c|}
\hline \multirow{3}{*}{$\begin{array}{l}\text { Song } \\
\text { character }\end{array}$} & \multicolumn{4}{|l|}{ Species } \\
\hline & \multicolumn{2}{|c|}{ D. montana } & \multicolumn{2}{|c|}{ D. littoralis } \\
\hline & $R$ & S.E. & $R$ & S.E. \\
\hline PN & 0.16 & 0.17 & - & \\
\hline PTL & 0.24 & 0.17 & - & \\
\hline PL & $0.85^{* * *}$ & 0.06 & 0.15 & 0.15 \\
\hline IPI & $0.79^{* * *}$ & 0.08 & $0.45^{* * *}$ & 0.13 \\
\hline $\mathrm{CN}$ & $0.85^{* * *}$ & 0.06 & $0.43^{* *}$ & 0.14 \\
\hline FRE & $0.45^{* *}$ & 0.16 & $0.86^{* * * *}$ & 0.05 \\
\hline
\end{tabular}

Significance tests for variation among individuals: ${ }^{* *} P<0.01,{ }^{* * *} P<0.001$.

\section{Means and variances}

The means and variances of the song characters of the wild-caught fathers and their sons raised in the laboratory were compared with each other to estimate the changes in phenotypic means and variation in songs due to a change in the environment. The rearing conditions affected the song characters in both species. The wild-caught $D$. montana males had, on average, longer sound pulses and fewer pulses in a pulse train than their sons (Table 2). The variance in most song characters was significantly larger among the fathers than among the offspring indicating that the natural environment is more variable than the controlled laboratory environment. 
In $D$. littoralis the mean PL was shorter among the fathers than among their offspring (Table 3). The sons also had less cycles in the pulse $(\mathrm{CN})$ and a lower basic frequency (FRE) than their fathers. Opposite to the expectations the phenotypic variances in song characters were generally larger among the offspring males than among the wild-caught males, the difference being significant in PL and FRE (Table 3 ).

The laboratory-reared males were larger than their wild-caught fathers in both species (Aspi, unpublished). Because in insects the songs of large males may differ from those of small males in various ways (e.g. Searcy \& Andersson, 1986), differences in means and variances could be the result of differences in body size in the two environments.

\section{Heritabilities}

In $D$. montana the estimates of heritabilities from father-son regression in the laboratory were rather high for most of the song characters, but not for FRE (Table 4). The heritability of PN appeared to be larger than the estimated repeatability and even larger than it's upper 95 per cent confidence limit $(0.52)$. In $D$.

Table 2 Mean and standard deviations of song characters in wild-caught males and in their $\mathrm{F}_{1}$-offspring in D. montana $(N=81)$. $t$ - and $F^{\prime}$-test values for PTL, IPI and PL are for log-transformed data

\begin{tabular}{lcccl}
\hline $\begin{array}{l}\text { Song } \\
\text { character }\end{array}$ & $\begin{array}{c}\text { Wild-caught } \\
\text { (Mean } \pm \text { s.d.) }\end{array}$ & $\begin{array}{l}\text { Offspring } \\
\text { (Mean } \pm \text { s.d.) }\end{array}$ & $t$ & $F^{\prime}$ \\
\hline PN & $8.3 \pm 0.8$ & $8.7 \pm 0.7$ & $-3.16^{* *}$ & 1.36 \\
PTL (ms) & $247.4 \pm 28.8$ & $255.3 \pm 27.8$ & -1.82 & 1.15 \\
IPI (ms) & $27.2 \pm 3.3$ & $26.6 \pm 2.0$ & 0.06 & 1.46 \\
PL $(\mathrm{ms})$ & $14.9 \pm 2.7$ & $13.9 \pm 2.2$ & $-2.42^{*}$ & $1.66^{*}$ \\
CN & $4.2 \pm 1.2$ & $4.2 \pm 0.9$ & 0.16 & $1.78^{*}$ \\
FRE (Hz) & $281.9 \pm 60.2$ & $298.0 \pm 39.1$ & -2.05 & $2.37^{* * *}$ \\
\hline
\end{tabular}

Significance tests: ${ }^{*} P<0.05,{ }^{* *} P<0.01,{ }^{* * *} P<001$.

Table 3 Mean and standard deviations of song characters in wild-caught males and in their $F_{1}$-offspring in $D$. littoralis $(N=85)$. $t$ - and $F^{\prime}$-test values for IPI and PL are for logtransformed data

\begin{tabular}{lccll}
\hline $\begin{array}{l}\text { Song } \\
\text { character }\end{array}$ & $\begin{array}{l}\text { Wild-caught } \\
\text { (Mean } \pm \text { s.d.) }\end{array}$ & $\begin{array}{l}\text { Offspring } \\
\text { (Mean } \pm \text { s.d.) }\end{array}$ & $t$ & $F^{\prime}$ \\
\hline IPI (ms) & $27.8 \pm 4.7$ & $27.6 \pm 3.7$ & 0.06 & 1.46 \\
PL (ms) & $48.1 \pm 5.4$ & $50.8 \pm 7.8$ & $2.42^{*}$ & $1.66^{*}$ \\
CN & $16.3 \pm 2.0$ & $14.4 \pm 2.5$ & $5.71^{* * *}$ & 1.49 \\
FRE (Hz) & $340.5 \pm 27.3$ & $286.7 \pm 52.3$ & $8.41^{* * *}$ & $3.65^{* * *}$ \\
\hline
\end{tabular}

Significance tests: ${ }^{*} P<0.05,{ }^{* * *} P<0.001$. littoralis none of the song characters showed significant heritable variation even under laboratory conditions (Table 5).

In $D$. montana the heritability estimates based on the across-environment regression were generally lower than the estimates measured for laboratoryreared flies (Table 4). However, the comparison of laboratory and across-environment regressions using the analysis of covariance revealed that differences between the two heritability estimates were significant only for PL $\left(F_{(1,134)}=6.13 ; P<0.05\right)$, and IPI $\left(F_{(1,134)}=5.35 ; P<0.05\right)$.

The estimate of $h_{\mathrm{V}}^{2}$ for PL and CN was close to the heritability estimate obtained from the across-environment regression. This indicates that $h_{(\mathrm{SL}-\mathrm{FN})}^{2}$ estimates are rather reliable (although non-significant) indicators of the heritability of these characters in the field, and that they are lower than $h_{(\mathrm{SL}-\mathrm{FL})}^{2}$ estimates is only due to larger phenotypic variation in the field. For the rest of the characters different heritability estimates were more dissimilar, suggesting possible genotype-environment interaction affecting also the variance components. The estimate of $h_{(\mathrm{SL}-\mathrm{FN})}^{2}$ for FRE appeared to underestimate the heritability in nature by an unknown amount and this estimate sets only the lower bound to the heritability suggesting larger additive genetic variance in the nature than in the laboratory. The across-environment heritability estimates for PTL, IPI and $\mathrm{PN}$ were overestimates and the minimum estimates for these characters were obtained using the formula provided Riska et al. (1989). Since already the regression coefficients of PTL and IPI were lower than zero, we could not reveal that these characters had additive genetic variation in the field. On the contrary, the significant across-environment regression for PN is sufficient evidence that the character is heritable in the field

Table 4 Heritability estimates for the song characters in $D$. montana in the laboratory $\left.\left(h_{\text {(SL-FL }}^{2}\right) ; N=56\right)$, across laboratory and natural environments $\left(h_{\mathrm{SL}-\mathrm{FN}}^{2}\right) ; N=81$ ), supposing additive genetic variance being similar in these two environments $\left(h_{V}^{2}\right)$, and the minimum estimate for the heritability in nature $\left(h_{\text {MIN }}^{2}\right)$

\begin{tabular}{lccrcc}
$\begin{array}{l}\text { Song } \\
\text { character }\end{array}$ & $h_{\text {(SL-FL) }}^{2} \pm$ S.E. & \multicolumn{1}{c}{$h_{(\mathrm{SL}-\mathrm{FN})}^{2} \pm$ S.E. } & \multicolumn{1}{l}{$h_{\mathrm{V}}^{2}$} & \multicolumn{1}{c}{$h_{\mathrm{MIN}}^{2}$} \\
\hline PN & $0.66^{*} \pm 0.25$ & $0.43^{*} \pm 0.19$ & 0.65 & 0.29 \\
PTL & $0.36 \pm 0.32$ & $-0.15 \pm 0.21$ & 0.36 & $<0$ \\
IPI & $0.80^{*} \pm 0.37$ & $-0.06 \pm 0.15$ & 0.38 & $<0$ \\
PL & $0.69^{*} \pm 0.27$ & $0.20 \pm 0.18$ & 0.23 & 0.17 \\
CN & $0.44 \pm 0.26$ & $0.13 \pm 0.17$ & 0.11 & 0.13 \\
FRE & $-0.23 \pm 0.34$ & $0.18 \pm 0.15$ & -0.04 & 0.18 \\
\hline
\end{tabular}

Significance of regression coefficient: ${ }^{*} P<0.05$. 
Table 5 Heritability estimates for the song characters in $D$. littoralis in the laboratory $\left(h_{\mathrm{SL}-\mathrm{FL}}^{2} ; N=60\right)$, across laboratory and natural environments $\left(h_{\mathrm{SL}-\mathrm{FL}}^{2}\right) ; N=85$ ), supposing additive genetic variance being similar in these two environments $\left(h_{\mathrm{V}}^{2}\right)$, and the minimum estimate for the heritability in nature $\left(h_{\text {MIN }}^{2}\right)$

\begin{tabular}{lrrrc}
\hline $\begin{array}{l}\text { Song } \\
\text { character }\end{array}$ & $h_{(\mathrm{SL} \cdot \mathrm{FL})}^{2} \pm$ S.E. & \multicolumn{1}{c}{$h_{(\mathrm{SL} \cdot \mathrm{FN})}^{2} \pm$ S.E. } & \multicolumn{1}{l}{$h_{\mathrm{V}}^{2}$} & \multicolumn{1}{c}{$h_{\mathrm{MIN}}^{2}$} \\
\hline IPI & $0.18 \pm 0.19$ & $0.15 \pm 0.18$ & 0.23 & 0.10 \\
PL & $0.15 \pm 0.25$ & $-0.04 \pm 0.28$ & 0.21 & $<0$ \\
CN & $-0.33 \pm 0.22$ & $0.00 \pm 0.22$ & -0.00 & $<0$ \\
FRE & $-0.02 \pm 0.23$ & $0.14 \pm 0.36$ & -0.02 & 0.14 \\
\hline
\end{tabular}

(Riska et al., 1989) although the magnitude of heritability is somewhat lower than the estimate based on this regression.

Since in the analysis of covariance there was significant difference between regression slopes in IPI and PL, and the across-environment heritability estimates appeared to be reliable or overestimates, it can be concluded that there were significant differences in heritabilities in the laboratory and in the field in these characters. Heritability estimates for PN and for $\mathrm{CN}$ were evidently not significantly different between the environments. Although the within-laboratory and the across-environment regressions for PTL and FRE were not significantly different, the real heritabilities in the laboratory and in the field may not be equal because the real difference between these heritabilities cannot be detected.

In $D$. littoralis the across-environment heritability estimates were of the same level (IPI) or even larger than in the laboratory (CN, FRE). Only for PL the regression slope across the environments was significantly lower $\left(F_{(1,142)}=14.07 ; P<0.001\right)$ than in the laboratory. Since the phenotypic variation in PL was larger among the laboratory reared offspring than among their wild-caught fathers, the larger environmental variation cannot be the reason for the lower across-environment heritability estimates. This indicates that the higher laboratory estimate of heritability is biased due to gene-environment interaction. Since the $h_{(\mathrm{SL-FN})}^{2}$ seems to overestimate the real heritability in the field, the results suggest that the heritabilities for this character differ in the laboratory and in the field. The $h_{(\mathrm{SL}-\mathrm{FL})}^{2}$ and $h_{\text {(SL-FN) }}^{2}$ estimates for IPI were almost equal and the slightly larger value of $h_{\mathrm{V}}^{2}$ is probably due only to larger phenotypic variation in the laboratory. The $h_{\text {(SL-FN) }}^{2}$ estimate for CN seems to be rather a reliable estimator for the heritability in the field (although zero), and thus it is not significantly different between environments. The $h_{\{\mathrm{SLFN}\rangle}^{2}$ estimate for FRE may be an underestimate, but given the large standard errors of the estimates for this character, the natural and laboratory estimates do not probably differ from each other.

\section{Discussion}

We have previously found (Aspi \& Hoikkala, in preparation) that $D$. montana and $D$. littoralis females probably use both PL and CN in male courtship song in their mate choice, the females of both species preferring those males with short and dense pulses in their songs. In $D$. montana the laboratory heritabilities of both of these characters were rather high, in $D$. littoralis the estimates were lower and for $\mathrm{CN}$ even negative. In both species the heritability estimates of PL were significantly lower in the field. The difference in heritabilities between the environments in D. montana was due to larger environmental variance in the field, but in D. littoralis interaction between genotype and environment was probable. We could not reveal that significant amounts of additive genetic variance exist in the sexually selected male song characters in either species in the field.

Although natural heritabilities for the characters preferred by the females had not been previously presented for insect species, several authors have estimated natural heritabilities of characters which are important in intermale competition. Body size in Drosophila melanogaster and D. buzzatii (e.g. Partridge et al., 1987; Taylor \& Kekić, 1988; Santos et al., 1988), and also the ability to hold territories in $D$. melanogaster (Hoffman \& Cacoyianni, 1989), have been shown to be important determinants of male mating success. Hoffman (1991) did not find evidence for heritable variation in territorial success in one or two of his natural populations of $D$. melanogaster, although he found additive genetic variance in this character among laboratory-reared flies. Hoffman (1991) also found heritable variation in body weight among the males of a laboratory stock, but not among wild-caught males, whose offspring were bred in the laboratory. In Coyne \& Beecham's (1987) study the male size of $D$. melanogaster was heritable in nature, but the heritability was significantly lower in the field than under laboratory conditions. The heritability of male thorax length in $D$. buzzatii has also been shown to be less in the wild than in the laboratory (Prout \& Barker, 1989). The differences between the environments in these studies have been partially due to larger environmental variation in the field (Prout \& Barker, 1989; Ruiz et al., 1991), but also due to genotype-environment interactions (Coyne \& Beecham, 1987; Hoffman, 1991).

Theoretical models of evolution of female choice consist of two groups of models (e.g. Bradbury \& Andersson, 1987). Functional 'good genes' models of 
sexual selection suggest that the preferred male character is a condition-dependent trait that reflects an individual's viability (e.g. Andersson, 1986; Iwasa et al., 1991), whereas in quantitative 'arbitrary trait' models (e.g. Lande, 1981) the male character itself is under weak stabilizing net selection [although the selection may even become disruptive depending about the variance of female preference (Lande, 1981; Barton \& Turelli, 1991)].

In empirical laboratory studies characters closely connected with fitness typically have very low heritabilities $(<0.2)$ (Falconer, 1981; Mousseau \& Roff, 1987; Roff \& Mousseau, 1987), presumably because continuous directional selection tends to exhaust additive genetic variability in fitness (Fisher, 1958; Falconer, 1981; Charlesworth, 1987; but see Price \& Schluter, 1991). Metric characters supposed to be subject to weak stabilizing selection (Bulmer, 1989) have had empirically substantial genetic variabilities with a typical heritability of 0.5 (Roff \& Mousseau, 1987; Mousseau \& Roff, 1987).

The previous laboratory estimates for heritabilities of the characters under female choice in insects have varied between 0.76 (Hedrick, 1988) and 0.32 (Carson $\&$ Lande, 1984). These heritabilities resemble in their magnitude the heritabilities of quantitative characters supposed to be under weak stabilizing selection rather than heritabilities of characters closely related to fitness. Only Butlin \& Hewitt's estimate (1986) of syllable length (character known to be under stabilizing sexual selection: Butlin et al., 1985) in the grasshopper, Chorthippus brunneus, has shown non-significant heritability $(-0.21$; Butlin \& Hewitt, 1986). However, there should be no additive genetic variation in selected traits independent of the mode of selection in evolutionary equilibrium (Fisher, 1958; Falconer, 1981; Charlesworth, 1987). As our results and other studies where the heritabilities have been measured both in the laboratory and in the field suggest, it may turn out that although sexually selected characters may be heritable in the laboratory, a much lower amount of additive genetic variability is maintained in the field due to larger environmental variation and genotype-environment interactions.

It seems, however, that in some cases substantial amounts of additive genetic variability can be maintained in sexually selected characters even in the field (e.g. Møller, 1989, 1991; Coyne \& Beecham, 1987; Hoffman, 1991), and some mechanism which may explain the observed additive genetic variance maintained in these characters is required. The observed low amount of genetic variability in fitness-related characters can be maintained by mutation-selection balance alone (Charlesworth, 1987; Rice, 1988), but it is not clear whether genetic variance can be maintained in characters under stabilizing selection. The previous mutation-selection models supposed that the balance between selection to deplete and mutation to restore genetic variability can also maintain the substantial amount of genetic variance and empirical heritabilities observed in characters under weak stabilizing selection (e.g. Lande, 1975), whereas latter models have doubted this as a probable mechanism in maintaining genetic variability (Turelli, 1984, 1985). The predictions of the models seem to be quite sensitive to assumptions about the underlying genetics (see Bulmer, 1989 for a review), but as Bulmer (1989) stated there may be 'problems in attributing the maintenance of genetic variability to mutation-selection balance'. Moreover, in some cases there is evidently a larger amount of additive genetic variance in characters under sexual selection (Møller, 1989, 1991) than can be maintained by mutation-selection balance alone, and other mechanisms are required for their maintenance. Probable alternatives to the mutation-selection balance for explaining the observed variability are balancing selection produced by pleiotropy (Rose, 1982; Barton, 1990), interactions between genotype and environment within natural habitats (e.g. Via \& Lande, 1987; Gillespie \& Turelli, 1989), multiplicative interactions between loci (Gimelfarb, 1989) and local mating in structured populations together with genetic redundancy (Goldstein \& Holsinger, 1992).

\section{Acknowledgements}

We thank Timothy S. Whittier, Jaakko Lumme and an anonymous referee for their useful comments on the manuscript.

\section{References}

ANDERSSON, M. B. 1986. Evolution of condition-dependent sex ornaments and mating preferences: sexual selection based on viability differences. Evolution, 40, 804-816.

ASP1, J., LUMME, J., HOIKKALA, A. AND HEIKKINEN, E. 1993. Reproductive ecology of the boreal riparian guild of Drosophila. Ecography, in press.

BARTON, N. H. 1990. Pleiotropic models of quantitative variation. Genetics, 124, 773-782.

BARTON, N. H. AND TURELli, M. 1991. Natural and sexual selection on many loci. Genetics, 127, 229-255.

BECKER, W. A. 1975. Manual of Quantitative Genetics, 3rd edn. Student Book Corporation, Washington.

BOGOY, T. P. AND BECKER, w. A. 1963. Exact confidence intervals for genetic heritability estimated from paternal half-sib correlations. Biometrics, 19, 494-496.

BRADBURY, J. W. AND ANDERSSON, M. B. (eds). 1987. Sexual Selection: Testing the Alternatives. Wiley, Chichester. 
BULMER, M. G. 1989. Maintenance of genetic variability by mutation-selection balance: a child's guide through the jungle. Genome, 31, 761-767.

BUTLIN, R. K., HEWITT, G. M. AND WEBB, S. F. 1985. Sexual selection for intermediate optimum in Chorthippus brunneus. Anim. Behav., 33, 1281-1292.

BUTLIN, R. K. AND HEWITT, G. M. 1986. Heritability estimates for characters under sexual selection in the grasshopper, Chorthippus brunneus. Anim. Behav., 34, 1256-1261.

CADE, w. H. 1981. Alternative male strategies: genetic differences in crickets. Science, 212, 563-564.

CARSON, H. L. AND LANDE, R. 1984. Inheritance of a secondary sexual character in Drosophila silvestris. Proc. Natl. Acad. Sci. U.S.A., 81, 6904-6907.

CHARLESWORTH, B. 1987. The heritability of fitness. In: Bradbury, J. W. and Andersson, M. B. (eds) Sexual Selection: Testing the Alternatives, Wiley, Chichester, pp. $23-40$.

COYNE, J. A. AND BEECHAM, E. 1987. Heritability of two morphological characters within and among populations of Drosophila melanogaster. Genetics, 117, 727-737.

FALCONER, D. S. 1981. Introduction to Quantitative Genetics, 2nd edn. Longman, Harlow.

FISHER, R. A. 1958. The Genetical Theory of Natural Selection, 2nd edn. Dover, New York.

GILLESPIE, J. H. AND TURELLI, M. 1989. Genotype-environment interactions and the maintenance of polygenic variation. Genetics, 121, 129-138.

GIMELFARB, A. 1989. Genotypic variation for a quantitative character maintained under stabilizing selection without mutations: epistasis. Genetics, 123, 27-227.

GOLDSTEIN, D. B. AND HOLSINGER, K. E. 1992. Maintenance of polygenic variation in spatially structured populations: roles of local mating and genetic redundancy. Evolution, 46, 412-429.

HEDRICK, A. V. 1988. Female choice and the heritability of attractive male traits: an empirical study. Am. Nat., 132, 267-276.

HOFFMAN, A. A. 1991. Heritable variation for territorial success in field-collected Drosophila melanogaster. Am. Nat., 138, 668-679.

HOFFMAN, A. A. AND CACOYIANN1, z. 1989. Territoriality in Drosophila melanogaster as a conditional strategy. Anim. Behav., 40, 526-537.

HolkKala, A., LAKovaARA, S. AND ROMPPAINEN, E. 1982. Mating behaviour and male courtship sounds in the Drosophila virilis group. In: Lakovaara, S. (ed.) Advances in Genetics, Development and Evolution of Drosophila, Plenum, New York, pp. 407-421.

IWASA, Y., POMIANKOWSKJ, A. AND NEE, S. 1991. The evolution of costly mate preference. II. The 'handicap principle'. Evolution, 45, 1431-1442.

LANDE, R. 1975. The maintenance of genetic variability by mutation in a polygenic character with linked loci. Genet. Res., 26, 221-235.

LANDE, R. 1981. Modes of speciation by sexual selection on polygenic traits. Proc. Natl. Acad. Sci. U.S.A., 78, 3721-3725.

MCLAIN, D. K. 1987. Heritability of size, a sexually selected character, and the response to sexual selection in a natural population of the southern green stink bug, Nezara viridula (Hemiptera: Pentatomidae). Heredity, 59, 391-395.

MOLlER, A. P. 1989. Natural and sexual selection on a plumage signal of status and on morphology in house sparrows. $J$. Evol. Biol., 2, 125-140.

MøLLER. A. P. 1991. Sexual selection in the monogamous barn swallow (Hirundo rustica). I. Determinants of tail ornament size. Evolution, 45, 1823-1836.

MOORE, A. J. 1990. The inheritance of social dominance, mating behaviour and attractiveness to mates in male Nauphoeta cinerea. Anim. Behav., 39, 388-397.

MOUSSEAU, T. A. AND ROFF, D. A. 1987. Natural selection and the heritability of fitness components. Heredity, 58, 181-197.

PARTRIDGE, L., HOFFMAN, A. A. AND JONES, J. S. 1987. Male size and mating success in Drosophila melanogaster and Drosophila pseudoobscura under field conditions. Anim. Behav., 35, 468-476.

PRICE, T. AND SCHLUTER, T. 1991. On the low heritability of lifehistory traits. Evolution, 45, 853-861.

PROUT, T. 1958. A possible difference in genetic variances between wild and laboratory populations. Dros. Inf. Serv., 32, 148-149.

PROUT, T. AND BARKER, J. S. F. 1989. Ecological aspects of the heritability of body size in Drosophila buzzatii. Genetics, 123, 803-813.

RICE, W. R. 1988. Heritable variation in fitness as a prerequisite for adaptive female choice: the effect of mutation-selection balance. Evolution, 42, 817-820.

RISKA, B., PROUT, T. AND TURELLI, M. 1989. Laboratory estimates of heritabilities and genetic correlations in nature. Genetics, 123, 865-871.

ROFF, D. A. AND MOUSSEAU, T. A. 1987. Quantitative genetics and fitness: lessons from Drosophila. Heredity, 58, 103-118.

ROSE, M. R. 1982. Antagonistic pleiotropy, dominance, and genetic variation. Heredity, 48, 63-78.

RUIZ, A., SANTOS, M., BARBADILla, A., QUEZEDA-DiAZ, J. E., HASSON, E. AND FONTDEVILA, A. 1991. Genetic variance for body size in a natural population of Drosophila buzzatii. Genetics, 128, 739-750.

SANTOS, M., RUIZ, A., BARBADILla, A., QUEZADA-DIAZ, J. E., HASSON, E. AND FONTDEvILA, A. 1988. The evolutionary history of Drosophila buzzatii: XIV. Larger flies mate more often in nature. Heredity, 61, 255-262.

SEARCY, W. A. AND ANDERSSON, M. 1986. Sexual selection and the evolution of song. Ann. Rev. Ecol. Syst., 17, 507-533.

TAYLOR, C. E. AND KEKIC, v. 1988. Sexual selection in a natural population of Drosophila melanogaster. Evolution, $\mathbf{4 2}$, 197-199.

TURELLI, M. 1984. Heritable genetic variation via mutation-selection balance: Lerch's zeta meet the abdominal bristle. Theor. Pop. Biol., 25, 138-193.

TURELL1, M. 1985. Effects of pleiotropy on predictions concerning mutation-selection balance for polygenic traits. Genetics, $111,165-195$.

VIA, S. AND LANDE, R. 1987. Evolution of genetic variability in a spatially heterogenous environment: effects of genotype-environment interaction. Genet. Res., 49, 147-156. 